\title{
Towards a Micropositioning System for Targeted Drug Delivery in Wireless Capsule Endoscopy
}

\author{
Stephen P. Woods and Timothy G. Constandinou, Senior Member, IEEE
}

\begin{abstract}
This paper describes a novel micropositioning mechanism for achieving $1 \mathrm{ml}$ of targeted drug delivery within wireless capsule endoscopes. The mechanism allows a needle to be positioned within a $22.5^{\circ}$ segment of a cylindrical capsule and be extendible by up to $4 \mathrm{~mm}$. The mechanism achieves both these functions using only a single micromotor and occupying a volume of just $200 \mathrm{~mm}^{3}$ (including micromotor), this represents only $6.6 \%$ of the total available space. Through a detailed stress analysis it has been shown that the proposed mechanism can be fabricated using FDA approved materials and requires a power budget of under $3.3 \%$ of the available capacity. It is envisaged this mechanism would empower a new breed of capsule microrobots for therapy in addition to diagnostics for pathologies such as ulcerative colitis and small intestinal Crohn's disease.
\end{abstract}

\section{INTRODUCTION}

The diagnosis and treatment of pathologies of the gastrointestinal (GI) tract are performed routinely by gastroenterologists using endoscopes and colonoscopes however the small intestinal tract is out of the reach of these conventional systems [1]. It is now commonplace for clinicians to access inside the small intestines using wireless pill-sized cameras which relay images of the intestinal wall for evaluation. This practice enables the detection and diagnosis of pathologies of the GI tract such as Crohn's disease, small intestinal tumours such as lymphoma and small intestinal cancer.

An early example of a basic pill-sized camera system is the M2A. The M2A is a swallowable wireless capsule endoscope (WCE) developed by Given Imaging Ltd. [2] in 2000 to overcome the problem of examining the small intestine. The capsule which is $11.0 \mathrm{~mm}$ in diameter and $25.0 \mathrm{~mm}$ long comprises a CMOS-based image sensor, four LEDs for illumination, an RF module for communication and an on-board power supply. Another similar system is the EndoCapsule by Olympus [3], which uses a CCD-based image sensor with 6 LEDs.

There is a clinical need to target and treat pathologies of the GI tract such as ulcerative colitis, polyps and Crohn's disease [4]. Current systems are in general limited to diagnostic use and do not have the ability to deliver therapy to the GI tract particularly within the confined space of the small intestine.

Resent measures have been taken to address this need through the application of regional drug absorption services.

The authors would like to thank Consultant Physician Nick Oliver for clinical insight and much useful discussion.

All authors are with the Centre for Bio-Inspired Technology, Department of Electrical and Electronic Engineering and Institute of Biomedical Engineering, Imperial College of Science, Technology and Medicine, London SW7 2AZ, United Kingdom. s. woods09@imperial.ac.uk

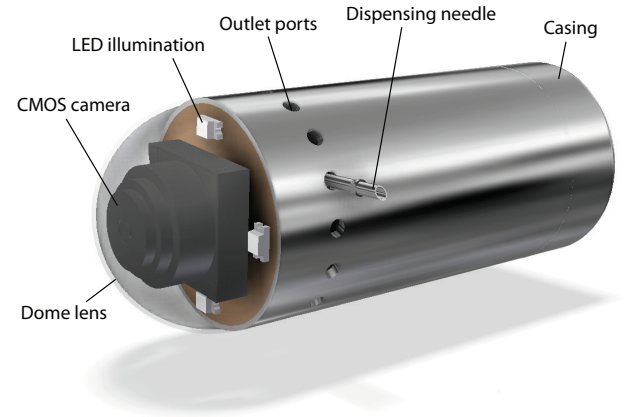

Fig. 1. Microrobot concept design capable of delivering targeted therapy to the gastrointestinal tract

The IntelliCap [5] by Philips Electronics, the InteliSite [6] by Innovative Devices LLC and the Enterion capsule [7] by Phaeton Research can all deliver medication to a region of the GI tract by means of releasing up to $1 \mathrm{ml}$ of medication which is stored onboard the capsule. The medication can be expelled to a target region of the GI tract such as the jejunum, ileum, ascending colon or descending colon.

Such systems are however unable to target specific pathogens such as tumours or ulcers because they release their payload progressively over a period of time or as a bolus form. This has the effect of spreading the medication over a section of lumen (due to peristalsis) as they have no means of stopping or holding their position.

This paper presents a novel micropositioning system capable of targeting and treating pathologies of the GI tract. Specifically this paper details the subminiature needle positioning and deployment mechanism (the stopping/holding mechanism is not within the scope of this paper). The paper is organised as follows: Section II describes the design requirements, Section III describes the adopted concept and Sections IV and V analyse the mechanics and conclude.

\section{DESIGN REQUIREMENTS}

The clinical need to target a specific location or feature within the GI tract for medication delivery can be achieved using a microrobot consisting of an onboard image sensor and a drug targeting system (based on propelling the medication through a needle). Fig. 1 shows a concept design of a microrobot with an integrated targeting system.

The proposed system would be more effective than current systems due to the advantages of the shorter discharge time and the more accurate delivery position. The shorter discharge time will allow for the correct dosage to be delivered 
to the required location without it being diluted over the delivery time from the natural passing of the GI tract or from being dispersed by the constant movement from peristalsis.

\section{A. Design challenges}

There are a number of design challenges that need to be addressed to develop a working prototype capable of being ingested. For example the limited capsule volume makes integrating actuator mechanism and energy storage devices particularly demanding:

1) Volume: Developing a targeting mechanism capable of manoeuvring a needle requires the application of microactuators however there are several limitations with current microactuators which prevent a simple system integration. Piezoelectric actuators require displacement amplification mechanisms to obtain a useful stroke length and also require large drive voltages (100V) [8]. Micromotors could potentially be used to drive mechanisms directly, however there are very few commercially available which could fit within the package size of the capsule and have a useful torque capability at a low RPM.

An alternative to micromotors as an actuator mechanism are shape memory alloys (SMA) such as superelastic nitinol which is an alloy of approximately 50\% nickel and 50\% titanium. The superelastic properties of nitinol allow large amounts of deformation to take place without permanently damaging the lattice structure of the material. This allowable strain makes it possible to create super springs which would be triggered by the Joules effect. The disadvantage of SMAs is their relatively poor efficiency, therefore thermal dissipation may lead to an elevated capsule temperature that is highly undesirable [9].

2) Torque: A crucial feature of micromotors is their ability to deliver a useful torque. It is possible to estimate the torque required to operate the targeting mechanism by using dimensions based on the $\mathrm{M} 2 \mathrm{~A}$ and applying equation $T=m \alpha k^{2}$. The radius of gyration $(k)$ would be $5.5 \mathrm{~mm}$, the angular acceleration $(\alpha)$ can be derived from the motor's performance, also by approximating the volume of steel the micromotor would be required to move, the mass $(\mathrm{m})$ of the steel can be found by rearranging equation $\rho=\mathrm{kg} / \mathrm{m}^{3}$.

The results of the initial torque analysis, which are based on using a $1.5 \mathrm{~mm}$ diameter $\times 10.5 \mathrm{~mm}$ long micromotor manufactured by Namiki (no. 10-010) show that a torque of $3.22 \mu \mathrm{Nm}$ would be required to operate the targeting mechanism. This figure is well within the $1.6 \mathrm{mNm}$ available from the Namiki motor however provision will need to be made for the inherent friction in the mechanism and for any load which would be generated by the needle positioning mechanism itself.

3) Energy requirement: One of the fundamental limitations of microactuators is power consumption as space to store energy is limited. Silver oxide button cell batteries are a standard method of powering WCEs with energy density ratios as high as $500 \mathrm{Wh} / L$ (watt hours per litre) however their median operating voltage is between 1.2 to
$1.5 \mathrm{~V}$ requiring at least two cells to directly power a $3.0 \mathrm{~V}$ micromotor.

Lithium Manganese Dioxide $\left(\mathrm{Li} / \mathrm{MnO}_{2}\right)$ batteries offer higher voltage ranges at 2.5 to $3.0 \mathrm{~V}$ with higher energy density ratios which can be as high as $550 \mathrm{Wh} / \mathrm{L}$ however they require a larger volume of space than lithium-ion polymer batteries (LiPo). LiPo batteries offer similar energy density ratios as lithium-ion batteries however their main advantage is their form factor.

\section{B. Target technical specification}

The size and shape of the microrobotic system are dependent on the limitations imposed by swallowing and on the microrobot's ability to navigate the ileocolic valve without becoming an obstruction. Determining the overall geometry presents a potential problem as the maximum volume a patient can swallow will vary greatly from person to person. A volume of $3.0 \mathrm{~cm}^{3}$ has been chosen as the maximum boundary for swallowing as recent research (Connor et al. 2009) has shown this to be achievable [10]. The target volume will be required to house all the components necessary to perform targeted therapy and microscale diagnosis. Table I lists the overall technical requirements for the microrobot platform.

TABLE I

OVERALL MICROROBOT TECHNICAL REQUIREMENTS

\begin{tabular}{lc}
\hline Requirement & Specification \\
\hline Microrobot volume & maximum $3.0 \mathrm{~cm}^{3}$ \\
Sensing & $\mathrm{pH}$, temp and pressure \\
Vision & $\mathrm{CMOS}$ and optical dome \\
Illumination & 4 white LEDs \\
Power source & onboard supply \\
Tracking & RF and time \\
Telemetry & bidirectional \\
Delivering therapy & liquid medication \\
Drug reservoir & $1 \mathrm{ml}$ \\
\hline
\end{tabular}

\section{DESIGN CONCEPT}

The needle positioning mechanism (Fig. 2) utilises a single micromotor to position and operate the needle. The design of the needle positioning mechanism consists of a $1.5 \mathrm{~mm}$ diameter $\times 10.5 \mathrm{~mm}$ long micromotor manufactured by Namiki, two opposing ratchets, a needle funnel, a needle cam and a movable needle. Also features integral to the microrobot's body are required. The first operation is the angular control of the position of the needle and the second is the extension and retraction of the needle. The medication will be stored in a compartment of the body and be delivered through the needle by means of pressure generated by a piston. The piston will be activated by a spring allowing for a rapid delivery of the medication.

It is a requirement that the needle can be positioned at selected points within a 360 degree envelope. For the purpose of prototyping 16 fixed positions equally spaced have been chosen. The angular positioning of the needle is achieved 


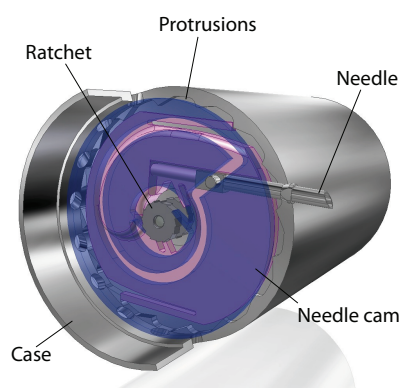

Fig. 2. Needle positioning mechanism assembly with material removed for clarity. Needle shown in the partially extended position

by the anticlockwise rotation of the micromotor while the advancement and retraction of the needle is achieved by the clockwise rotation of the micromotor.

\section{A. Positioning the needle}

Manoeuvring the needle to a selected position is achieved by applying a negative voltage to the micromotor. The negative voltage will cause the micromotor to rotate in an anticlockwise direction. The needle funnel (Fig. 3a) rotates anticlockwise by virtue of a ratchet which is mounted on the micromotor's driveshaft. The ratchet engages with a set of sprung legs which are integral to the needle funnel (Fig. 3b). The needle is engaged with the needle funnel and is carried round with it.

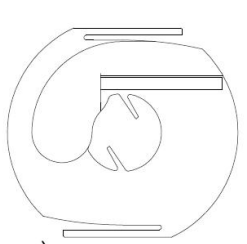

a)
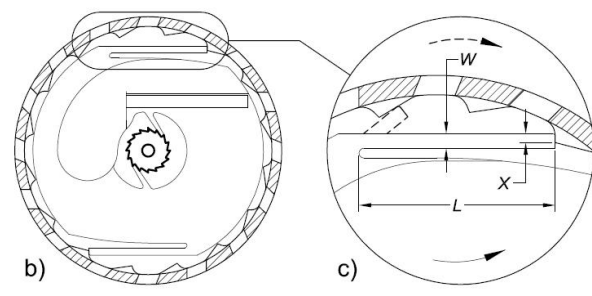

Fig. 3. Needle positioning mechanism extended cantilever: needle funnel a), ratchet driving the needle funnel b) and the needle funnel arm engaged with a protrusion $\mathrm{c}$ )

When the needle funnel is rotating anticlockwise an arm which protrudes from the side of the needle funnel rides over the top of a plurality of protrusions (Fig. 3b) on the inside face of the body. The function of these protrusions is to prevent the needle funnel from rotating clockwise when the motor is reversed. It achieves this by engaging the end of the arms with a parallel surface of the protrusion (Fig. 3c) preventing any further movement clockwise and aligning the needle with one of the 16 fixed ports on the microrobot's body.

\section{B. Operating the needle}

Applying a positive voltage to the micromotor reverses its direction, this disengages the needle funnel ratchet from its sprung legs and engages the needle cam ratchet with a set of sprung legs that are integral to the needle cam (Fig. 4a).

When engaged the ratchet drives the needle cam in a clockwise direction. The needle is engaged with a track in the
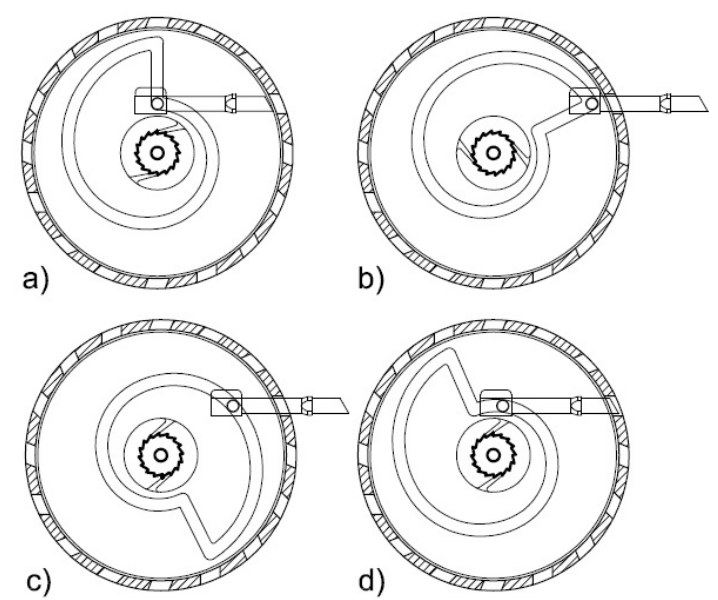

Fig. 4. 360 degree needle cam operating cycle: stored position a), full stroke b), needle returning to the stored position c), and retracted position d)

needle cam by means of a driving peg mounted on the side of the needle. The track is shaped to convert the rotational motion of the cam into a variable linear motion. This linear motion allows the advancement and retraction of the needle.

Fig. 4 shows the sequence of operation for the extension and retraction of the needle. Fig. 4a shows the needle in the stored position, this is the position the needle will be in when the microrobot will be swallowed and travel through the GI tract to the target site. Fig. $4 \mathrm{~b}$ shows the needle at full extension giving a reach of approximately $8.85 \mathrm{~mm}$ radius and a stroke length of $4.0 \mathrm{~mm}$. The design of the cam utilises a direct linear movement so that a positive force can be delivered to the needle which will be required to penetrate the wall of the GI tract. Fig. 4c shows the needle retracting backwards by virtue of the track slowly spiralling inwards. Fig. $4 \mathrm{~d}$ shows the needle returning to the stored position once the medication has been delivered.

\section{Design Analysis}

When the needle funnel (Fig. 3a) rotates anticlockwise the arm on the side is required to flex out of position as it rides over the protrusions on the body (Fig. 3c). This flexing out of position will result in a stress induced in the arm. The amount of stress in the arm will be a result of the material's geometry, the Young's modulus for the material and the amount of movement the arm will make. Various materials can be evaluated for their ability to withstand this stress and continue to function correctly. To determine the most suitable for this application Table II shows a selection of materials and their properties which can be compared for the purpose of evaluating the needle positioning arm (Fig. 3a).

Using the principle of a cantilevered beam the displacement of the beam can be calculated by using $\delta=P L^{3} / 3 E I$. As the distance the arm will be flexed through is known $(\delta)$ and the material's Young's modulus $E$, the second moment of area of the beam $I$ and the length of the arm $L$ are also known the equation can be rearranged to find the load (P) 
TABLE II

MATERIAL SELECTION MATRIX

\begin{tabular}{lccccc}
\hline Material & $\begin{array}{c}\text { Density } \\
\mathrm{g} / \mathrm{cm}^{3}\end{array}$ & $\begin{array}{c}\text { Yield } \\
\text { point } \\
\mathrm{N} / \mathrm{mm}^{2}\end{array}$ & $\begin{array}{c}\text { Shear } \\
\text { modulus } \\
k N / \mathrm{mm}^{2}\end{array}$ & $\begin{array}{c}\text { Young's } \\
\text { modulus } \\
k N / \mathrm{mm}^{2}\end{array}$ & $\begin{array}{c}\text { Poisson's } \\
\text { ratio }\end{array}$ \\
\hline Stainless Steel 304 & 8.08 & 290 & 72.4 & 193 & 0.3 \\
Ti Alloy 6AL 4V & 4.43 & 880 & 42.7 & 114 & 0.3 \\
Nylon 6 & 1.14 & 82 & 1.24 & 3.3 & 0.4 \\
PVC & 1.44 & 58 & 1.13 & 3.0 & 0.4 \\
Acetal Copolymer & 1.41 & 60 & 1.01 & 2.7 & 0.4 \\
PEEK 450G & 1.32 & 97 & 1.31 & 3.5 & 0.4 \\
\hline
\end{tabular}

that would be required to make the displacement. The applied load can be used to determine the stress induced in the arm.

\section{A. Needle funnel arm simulation}

For a structure to be considered stable in operation the maximum stress induced in the structure must be below the limiting property factor of strength for the material, this limit is known as the yield point of the material and it is the point where permanent deformation of the material occurs. Using the geometry of the needle funnel arm and a point load $(\mathrm{P})$ of $12.55 \mathrm{mN}$ derived from a $0.17 \mathrm{~mm}$ displacement of a PEEK needle funnel arm a finite element analysis (FEA) has been performed, Fig. 5.

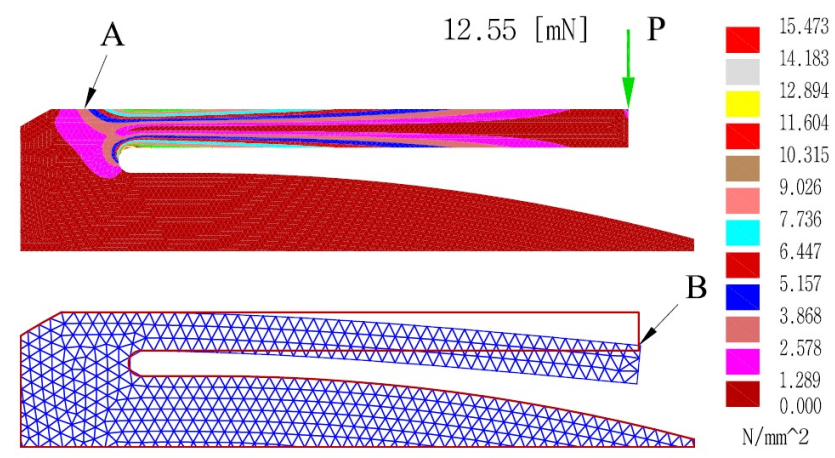

Fig. 5. Von Mises FEA 2D isoareas and displacement analysis of a PEEK needle funnel arm with a point load of $12.55 \mathrm{mN}$

The FEA analysis (Fig. 5) shows the maximum Von Mises stress to be $15.47 \mathrm{~N} / \mathrm{mm}^{2}$ from an applied load of $12.55 \mathrm{mN}$ at the end of the arm, also a stress of $3.86 \mathrm{~N} / \mathrm{mm}^{2}$ can be seen running through the beam as a contour of light brown indicated by arrow (A). The analysis shows that the maximum predicted stress is 6.25 times lower than the $97 \mathrm{~N} / \mathrm{mm}^{2}$ yield point of PEEK, this gives an acceptable margin for safety to accommodate for any variations in material and geometry. The displacement analysis, arrow (B), shows the movement of the arm. The resulting stress in the arm is of a magnitude which would allow the arm to recover back to its original starting position when the applied load is removed. This will allow the needle funnel to lock into position when it is rotated clockwise.

\section{B. Energy requirements}

It is an important requirement that the needle positioning mechanism can operate with low power consumption and as such the FEA findings have been used to reduce the micromotor's operating load. It has been calculated using a battery volume of $188 \mathrm{~mm}^{3}$ and a Lithium Manganese Dioxide battery that a complete cycle of the needle positioning mechanism will consume at most $12.16 \mathrm{~J}$ of the available energy. This is based on the peak power consumption of a $1.5 \mathrm{~mm}$ diameter $\times 10.5 \mathrm{~mm}$ long four stage geared micromotor manufactured by Namiki (no. 10-010) operating for approximately 2.5 seconds. This represents only $3.3 \%$ of the available energy, offering the potential for multiple repositions.

\section{CONCLUSION}

In this paper we have presented a novel mechanism towards the realisation of a practical micropositioning system for targeted drug delivery in the small intestines. The system can be used for the detection and treatment of pathologies of the GI tract such as Crohn's disease, small intestinal tumours such as lymphoma and small intestinal cancer.

It has been shown that the proposed needle positioning system achieves the required functionality whilst occupying a volume of only $200 \mathrm{~mm}^{3}$. This is based on a single micromotor and is estimated to consume a maximum of $12.16 \mathrm{~J}$ of energy per activation.

In order to achieve the control necessary to target a particular pathogen within the GI tract the microrobot would be required to hold itself in position, resisting the natural movement from peristalsis. It is envisaged that a stopping mechanism capable of resisting peristalsis can be incorporated into the standard geometry of the WCE, with the combined needle positioning system and stopping mechanism occupying $13 \%$ of the total available volume.

\section{REFERENCES}

[1] K.-C. Kong, J. Cha, D. Jeon and D.-I. Dan Cho. A rotational micro biopsy device for the capsule endoscope. IEEE/RSJ Int. Conf. Intellig. Robots Syst., pages 1839-1843, 2005.

[2] G. D. Meron. The development of the swallowable video capsule (M2A). Gastrointestinal End., 52:817-819, 2000.

[3] D. R. Cave et al. A multicenter randomized comparison of the endocapsule and the pillcam sb. GI End., pages 487-494, 2008.

[4] N. I. Goldfarb et al. Diagnosing crohn's disease: An economic analysis comparing wireless capsule endoscopy with traditional diagnostic procedures. Disease Management, 7:292-304, 2004.

[5] A. Forgione. In vivo microrobots for natural orifice transluminal surgery. current status and future perspectives. Surgical Oncol., 18:121-129, 2009.

[6] I. Wilding, P. Hirst and A. Connor. Development of a new engineeringbased capsule for human drug absorption studies. Pharma. Science \& Tech. Today, 3:385-392, 2000.

[7] P. J. Houzego, et al. Ingestible device for the release of substances at distinct locations in the alimentary canal, USA: Phaeton Research Ltd, 2005.

[8] J. Peirs, D. Reynaerts, and H. Van Brussel. Design of miniature parallel manipulators for integration in a self-propelling endoscope. Sensors and Actuators A: Physical, 85:409-417, 2000.

[9] S. Gorini, et al. A novel SMA-based actuator for a legged endoscopic capsule. IEEE/RAS-EMBS Int. Conf. Biomed. Robotics and Biomechatr, pages 443-449, 2006.

[10] A. Connor, et al. An oral human drug absorption study to assess the impact of site of delivery on the bioavailability of bevirimat. J Clin Pharmacol, 49:606-612, 2009. 